\title{
Approximation by regular functions in Sobolev spaces arising from doubly elliptic problems
}

\author{
Patrizia Pucci · Enzo Vitillaro \\ To the memory of our dear friend Professor Domenico Candeloro with high feelings \\ of admiration for his notable contributions in Mathematics
}

Received: date / Accepted: date

Abstract The paper deals with a nontrivial density result for $C^{m}(\bar{\Omega})$ functions, with $m \in \mathbb{N} \cup\{\infty\}$, in the space

$$
W^{k, \ell, p}(\Omega ; \Gamma)=\left\{u \in W^{k, p}(\Omega): u_{\mid \Gamma} \in W^{\ell, p}(\Gamma)\right\},
$$

endowed with the norm of $\left(u, u_{\mid \Gamma}\right)$ in $W^{k, p}(\Omega) \times W^{\ell, p}(\Gamma)$, where $\Omega$ is a bounded open subset of $\mathbb{R}^{N}, N \geq 2$, with boundary $\Gamma$ of class $C^{m}, k \leq \ell \leq m$ and $1 \leq p<\infty$.

Such a result is of interest when dealing with doubly elliptic problems involving two elliptic operators, one in $\Omega$ and the other on $\Gamma$.

Moreover we shall also consider the case when a Dirichlet homogeneous boundary condition is imposed on a relatively open part of $\Gamma$ and, as a preliminary step, we shall prove an analogous result when either $\Omega=\mathbb{R}^{N}$ or $\Omega=\mathbb{R}_{+}^{N}$ and $\Gamma=\partial \mathbb{R}_{+}^{N}$.

\begin{abstract}
The authors are members of the Gruppo Nazionale per l'Analisi Matematica, la Probabilità e le loro Applicazioni (GNAMPA) of the Istituto Nazionale di Alta Matematica (INdAM). The manuscript was realized within the auspices of the INdAM - GNAMPA Projects Equazioni alle derivate parziali: problemi e modelli (Prot_U-UFMBAZ-2020-000761). The first author was also partly supported by the Fondo Ricerca di Base di Ateneo - Esercizio 2017-2019 of the University of Perugia, named PDEs and Nonlinear Analysis, while the second author by the Progetto Equazione delle onde con condizioni acustiche, finanziato con il Fondo Ricerca di Base, 2019, della Università degli Studi di Perugia and by Progetti Equazioni delle onde con condizioni iperboliche ed acustiche al bordo, finanziati con i Fondi Ricerca di Base 2017 and 2018, della Università degli Studi di Perugia.
\end{abstract}

\section{P. Pucci}

Dipartimento di Matematica e Informatica, Università degli Studi di Perugia

Via Vanvitelli 1, 06123 Perugia, Italy

Tel.: +390755855038

Fax: +390755855024

E-mail: patrizia.pucci@unipg.it

E. Vitillaro

Dipartimento di Matematica e Informatica, Università degli Studi di Perugia

Via Vanvitelli 1, 06123 Perugia, Italy

Tel: +390755855015

Fax: +390755855024

E-mail: enzo.vitillaro@unipg.it 
Keywords Density results $\cdot$ Sobolev spaces - Smooth functions - the LaplaceBeltrami operator

Mathematics Subject Classification (2010) $46 \mathrm{E} 35 \cdot 46.38 \cdot 46 \mathrm{M} 35$

\section{Introduction and main results}

Density results for smooth functions in Sobolev spaces constitute a cornerstone in the classical theory of these spaces and in their applications in PDEs theory. Actually every textbook dealing with Sobolev spaces or PDEs devotes some attention to this subject, see for example [1,2,5,11,12,13, 14, 16, 17]. The paper deals with Sobolev spaces of integer nonnegative order, which are the most classical ones, but the density subject is standard also when working with Sobolev spaces of fractional order, also known as Sobolev - Slobodeckij spaces, and with Besov spaces and Bessel - potential ones. See [17].

When considering Sobolev spaces in $\mathbb{R}^{N}, N \geq 1$, it is well-known that the space of compactly supported smooth functions $C_{c}^{\infty}\left(\mathbb{R}^{N}\right)$ is dense in $W^{k, p}\left(\mathbb{R}^{N}\right)$ for $k \in \mathbb{N}$ and $1 \leq p<\infty$. Since the proof of this result relies on Friedrichs mollifiers and truncation arguments, following [4], we shall refer to this result as to Friedrichs' Theorem. When considering the same spaces in an open subset $\Omega$ of $\mathbb{R}^{N}, N \geq 1$, the same arguments show that $\left\{\left.u\right|_{\Omega}: u \in C_{c}^{\infty}\left(\mathbb{R}^{N}\right)\right\}$ is dense in $W^{k, p}(\Omega)$ for $k \in \mathbb{N}$ and $1 \leq p<\infty$, provided $\Gamma=\partial \Omega$ is continuous (see [11, Theorem 11.35, p. 330]).

Moreover, the celebrated Meyers Serrin Theorem (see [15]) asserts that $C^{\infty}(\Omega) \cap$ $W^{k, p}(\Omega)$ is dense in $W^{k, p}(\Omega)$, for $k \in \mathbb{N}$ and $1 \leq p<\infty$, without any regularity assumption on $\Gamma$. Also this result is treated in most textbooks in Sobolev spaces, see for example [11] and [20].

In the present paper we shall deal with a Friedrichs' type result. Indeed, as we are going to explain in the sequel, boundary regularity is needed even to state our first main result.

It is mathematical folklore that all density results for smooth functions in Sobolev spaces and in their closed subspaces can be trivially derived by the classical results stated above. On the other hand, for non-closed subspaces, the situation may be different. We refer here to [10], where the authors deal with the doubly elliptic problem

$$
\begin{cases}-\Delta u=f & \text { in } \Omega, \\ \partial_{v} u+\alpha u-\beta \Delta_{\Gamma} u=h & \text { on } \Gamma,\end{cases}
$$

where $\Omega \subset \mathbb{R}^{N}, N \geq 2$, is a bounded domain with $C^{m}$ boundary $\Gamma, m \in \mathbb{N} \cup\{\infty\}$, $\alpha, \beta$ are positive constants, $\nu$ denotes the outward normal to $\Omega$ and $\Delta_{\Gamma}$ stands for the Laplace-Beltrami operator on $\Gamma$. In [10, Remark 2.6] the authors realized that the density of $\left\{\left.u\right|_{\Omega}: u \in C^{m}\left(\mathbb{R}^{N}\right)\right\}$ in the space

$$
H^{m}(\Omega ; \Gamma)=\left\{u \in H^{m}(\Omega): u_{\mid \Gamma} \in H^{m}(\Gamma)\right\},
$$

endowed with standard product norm of the couple $\left(u, u_{\mid \Gamma}\right)$ in $H^{m}(\Omega) \times H^{m}(\Gamma)$, is nontrivial at all. Here and in the sequel $u \mapsto u_{\mid \Gamma}$ denotes the Trace Operator. See also [12, Lecture 12]. 
The same type of remark was made by the second author of the present paper in [18, 19] when dealing with a nonlinear perturbation of the problem

$$
\begin{cases}u_{t t}-\Delta u=0 & \text { in }(0, \infty) \times \Omega, \\ u=0 & \text { on }(0, \infty) \times \Gamma_{0}, \\ u_{t t}+\partial_{v} u-\Delta_{\Gamma} u=0 & \text { on }(0, \infty) \times \Gamma_{1}, \\ u(0, x)=u_{0}(x), \quad u_{t}(0, x)=u_{1}(x) & \text { in } \bar{\Omega},\end{cases}
$$

where $\Gamma$ is of class $C^{1}, \Gamma=\Gamma_{0} \cup \Gamma_{1}$ with $\overline{\Gamma_{0}} \cap \overline{\Gamma_{1}}=\emptyset$ and $\Gamma_{1} \neq \emptyset$. In particular the density of $\left\{\left.u\right|_{\Omega}: u \in C^{1}\left(\mathbb{R}^{N}\right), u=0\right.$ on $\left.\Gamma_{0}\right\}$ in the space

$$
H_{\Gamma_{0}}^{1}(\Omega ; \Gamma)=\left\{u \in H^{1}(\Omega): u_{\mid \Gamma} \in H^{1}(\Gamma), u_{\mid \Gamma}=0 \quad \text { on } \Gamma_{0}\right\}
$$

endowed with standard product norm of the couple $\left(u, u_{\mid \Gamma}\right)$ in $H^{1}(\Omega) \times H^{1}(\Gamma)$, was remarked to be nontrivial.

Clearly the two results above are particular cases of a slightly more general one. Before formulating it we remark that the space $H^{m}(\Gamma)$ appearing in (1) and in (2) (when $m=1$ ) is properly defined, through local charts, only when $\Gamma$ is at least $C^{m-1,1}$ (see [9]).

In this paper we shall take $\Gamma$ of class $C^{m}$ for the sake of simplicity and thus we shall consider $\Omega$ satisfying the following assumption:

(H) $\Omega$ is a bounded open subset of $\mathbb{R}^{N}, N \geq 2$, with $\Gamma=\partial \Omega$ of class $C^{m}, m \in \mathbb{N} \cup\{\infty\}$. Moreover $\Gamma=\Gamma_{0} \cup \Gamma_{1}, \overline{\Gamma_{0}} \cap \overline{\Gamma_{1}}=\emptyset$ and $\Gamma_{1} \neq \emptyset$.

We shall consider, for $k, \ell \in \mathbb{N}$ and $p \in[1, \infty)$, the Banach space

$$
W_{\Gamma_{0}}^{k, \ell, p}(\Omega ; \Gamma)=\left\{u \in W^{k, p}(\Omega): u_{\mid \Gamma} \in W^{\ell, p}(\Gamma): u_{\mid \Gamma}=0 \text { on } \Gamma_{0}\right\}
$$

with the standard contraction $W_{\Gamma_{0}}^{k, \ell, p}(\Omega ; \Gamma)=W^{k, \ell, p}(\Omega ; \Gamma)$ when $\Gamma_{0}$ is empty.

Clearly this space is of some interest only when $k \leq \ell$, since when $\ell<k$ it reduces to $W^{k, p}(\Omega)$ by the Trace Theorem. In the sequel we shall take $k \leq \ell \leq m$ and we shall identify $W_{\Gamma_{0}}^{k, \ell, p}(\Omega ; \Gamma)$ with its isometric copy

$$
W_{\Gamma_{0}}^{k, \ell, p}(\Omega ; \Gamma)=\left\{(u, v) \in W^{k, p}(\Omega) \times W^{\ell, p}(\Gamma): v=u_{\mid \Gamma}, v=0 \text { on } \Gamma_{0}\right\} .
$$

We shall endow it with the norm inherited from the product space. Let us remark that the case $\Gamma_{0}=\emptyset$ is also included in our treatment. The first main result is

Theorem 1 If assumption $(H)$ holds then

$$
Y=\left\{\left.u\right|_{\Omega}: u \in C_{c}^{m}\left(\mathbb{R}^{N}\right), u=0 \text { on } \Gamma_{0}\right\}
$$

is dense in $W_{\Gamma_{0}}^{k, \ell, p}(\Omega ; \Gamma)$ for all $k, \ell \in \mathbb{N}$, with $k \leq \ell \leq m$, and any $p \in[1, \infty)$. 
The proof of Theorem 1 relies on the combination of the standard localization technique with an analogous result in the case $\Omega=\mathbb{R}^{N}, \Gamma_{0}=\emptyset$ and $\Gamma_{1}=\partial \mathbb{R}_{+}^{N}$. Since this result could be of some independent interest we shall state here as our second main result. To state it we introduce the standard notation $x=\left(x^{\prime}, x_{N}\right) \in \mathbb{R}^{\mathbb{N}}$, with $x^{\prime} \in \mathbb{R}^{N-1}$ and $x_{N} \in \mathbb{R}$,

$$
\begin{gathered}
B_{r}\left(\mathbb{R}^{N-1}\right)=\left\{x^{\prime} \in \mathbb{R}^{N-1}:\left|x^{\prime}\right|<r\right\} \quad \text { for any } r>0, \\
\mathbb{R}_{+}^{N}=\left\{x=\left(x^{\prime}, x_{N}\right) \in \mathbb{R}^{N}: x_{N}>0\right\}, \quad \partial \mathbb{R}_{+}^{N}=\left\{x=\left(x^{\prime}, 0\right) \in \mathbb{R}^{N}: x^{\prime} \in \mathbb{R}^{N-1}\right\},
\end{gathered}
$$

and for $k, \ell \in \mathbb{N}$ and $p \in[1, \infty)$, the Banach space

$$
W^{k, \ell, p}\left(\mathbb{R}^{N} ; \partial \mathbb{R}_{+}^{N}\right)=\left\{u \in W^{k, p}\left(\mathbb{R}^{N}\right): u_{\mid \partial \mathbb{R}_{+}^{N}} \in W^{\ell, p}\left(\partial \mathbb{R}_{+}^{N}\right)\right\},
$$

where $W^{\ell, p}\left(\partial \mathbb{R}_{+}^{N}\right)$ is naturally identified with $W^{\ell, p}\left(\mathbb{R}^{N-1}\right)$. According to the previous identification, we shall also identify it with

$$
W^{k, \ell, p}\left(\mathbb{R}^{N} ; \partial \mathbb{R}_{+}^{N}\right)=\left\{(u, v) \in W^{k, p}\left(\mathbb{R}^{N}\right) \times W^{\ell, p}\left(\partial \mathbb{R}_{+}^{N}\right): v=u_{\mid \partial \mathbb{R}_{+}^{N}}\right\},
$$

and we shall endow it with the norm inherited from the product space. Also in this case only the case $k \leq \ell$ is of some interest. The second main result is

Theorem 2 Let $k, \ell \in \mathbb{N}$ and $p \in[1, \infty)$. For any $u \in W^{k, \ell, p}\left(\mathbb{R}^{N} ; \partial \mathbb{R}_{+}^{N}\right)$ there exists a sequence $\left(u_{n}\right)_{n}$ in $W^{k, \ell, p}\left(\mathbb{R}^{N} ; \partial \mathbb{R}_{+}^{N}\right) \cap C^{\infty}\left(\mathbb{R}^{N}\right)$ such that $\operatorname{supp} u_{n} \subseteq \operatorname{supp} u+\overline{B_{1 / n}\left(\mathbb{R}^{N}\right)}$ for all $n$ and $u_{n} \rightarrow$ u in $W^{k, \ell, p}\left(\mathbb{R}^{N} ; \partial \mathbb{R}_{+}^{N}\right)$.

Since Theorem 2 does not look as the exact translation of Theorem 1 in the case $\Omega=\mathbb{R}^{N}$, we would like to remark a trivial consequence of it. To state it we set, for $k$, $\ell \in \mathbb{N}$ and $p \in[1, \infty)$, the Banach space

$$
W^{k, \ell, p}\left(\mathbb{R}_{+}^{N} ; \partial \mathbb{R}_{+}^{N}\right)=\left\{u \in W^{k, p}\left(\mathbb{R}_{+}^{N}\right): u_{\mid \partial \mathbb{R}_{+}^{N}} \in W^{\ell, p}\left(\partial \mathbb{R}_{+}^{N}\right)\right\}
$$

endowed with the norm of the couple $\left(u, u_{\mid \partial \mathbb{R}_{+}^{N}}\right)$ in the product space. Since any element of $W^{k, p}\left(\mathbb{R}_{+}^{N}\right)$ possesses an extension in $W^{k, p}\left(\mathbb{R}^{N}\right)$ (see for example [1, Theorem 5.19 p.148]), by Theorem 2 we immediately get the next result.

Corollary 1 Let $k, \ell \in \mathbb{N}$ and $p \in[1, \infty)$. Then

$$
\left\{\left.u\right|_{\mathbb{R}_{+}^{N}}: u \in C^{\infty}\left(\mathbb{R}^{N}\right)\right\} \cap W^{k, \ell, p}\left(\mathbb{R}_{+}^{N} ; \partial \mathbb{R}_{+}^{N}\right)
$$

is dense in $W^{k, \ell, p}\left(\mathbb{R}_{+}^{N} ; \partial \mathbb{R}_{+}^{N}\right)$.

The proof of Theorem 2 is based on identifying the space $W^{k, p}\left(\mathbb{R}^{N}\right)$ with its vectorial version

$$
X^{k, p}=\bigcap_{j=0}^{k} W^{j, p}\left(\mathbb{R} ; W^{k-j}\left(\mathbb{R}^{N-1}\right)\right),
$$

on choosing mollifiers in separate form in the variables $x^{\prime} \in \mathbb{R}^{N-1}$ and $x_{N} \in \mathbb{R}$ and appropriately selecting their support radii.

Since a similar identification does not look to be trivial for non integer values of $k$, the extension of Theorems 1 and 2 to non integer values of $k$ and $\ell$ is not immediate. In the next section we are going to give the proofs of Theorems 12 


\section{Proofs}

Proof of Theorem 2 Let $\left(\rho_{n}^{\prime}\right)_{n}$ and $\left(\rho_{n}^{\prime \prime}\right)_{n}$ be two sequences of standard mollifiers in $\mathbb{R}^{N-1}$ and in $\mathbb{R}$, respectively. That is

$$
\begin{aligned}
& \rho_{n}^{\prime} \in C_{c}^{\infty}\left(\mathbb{R}^{N-1}\right), \quad \operatorname{supp} \rho_{n}^{\prime} \subseteq \overline{B_{1 / n}\left(\mathbb{R}^{N-1}\right)}, \quad \int_{\mathbb{R}^{N-1}} \rho_{n}^{\prime} d x^{\prime}=1, \quad \rho_{n}^{\prime} \geq 0 \text { in } \mathbb{R}^{N-1}, \\
& \rho_{n}^{\prime \prime} \in C_{c}^{\infty}(\mathbb{R}), \quad \operatorname{supp} \rho_{n}^{\prime \prime} \subseteq[-1 / n, 1 / n], \quad \int_{\mathbb{R}} \rho_{n}^{\prime \prime} d x_{N}=1, \quad \rho_{n}^{\prime \prime} \geq 0 \text { in } \mathbb{R} .
\end{aligned}
$$

Let $\left(\rho_{m, n}\right)_{m, n}$ be the double sequence of smooth functions in $\mathbb{R}^{N}$ defined in the separate form

$$
\rho_{m, n}(x)=\rho_{m}^{\prime}\left(x^{\prime}\right) \rho_{n}^{\prime \prime}\left(x_{N}\right) \text { for } x=\left(x^{\prime}, x_{N}\right) \in \mathbb{R}^{N} .
$$

Thus, for any couple of strictly increasing sequences $\left(\sigma_{n}\right)_{n},\left(\tau_{n}\right)_{n}$ in $\mathbb{N}$ such that $\sigma_{n}, \tau_{n} \geq 2 n$ for all $n$ the sequence $\left(\rho_{n}\right)_{n}$, with $\rho_{n}=\rho_{\sigma_{n}, \tau_{n}}$, is a standard mollifying sequence in $\mathbb{R}^{N}$.

Fix $u \in W^{k, \ell, p}\left(\mathbb{R}^{N} ; \partial \mathbb{R}_{+}^{N}\right)$ and a couple of strictly increasing sequences $\left(\sigma_{n}\right)_{n}$, $\left(\tau_{n}\right)_{n}$ in $\mathbb{N}$ such that $\sigma_{n}, \tau_{n} \geq 2 n$ for all $n$, which we select lately. Put $u_{m, n}=\rho_{m, n} *$ $u$. Standard properties of convolution and mollifiers, cf. Propositions 4.18 and 4.20 of [5], show that $\operatorname{supp} u_{\sigma_{n}, \tau_{n}} \subseteq \operatorname{supp} u+\overline{B_{1 / n}\left(\mathbb{R}^{N}\right)}$ since $\sigma_{n}, \tau_{n} \geq 2 n$ for all $n$, and $u_{\sigma_{n}, \tau_{n}} \in C^{\infty}\left(\mathbb{R}^{N}\right)$. Moreover, $u_{\sigma_{n}, \tau_{n}} \rightarrow u$ in $W^{k, \ell, p}\left(\mathbb{R}^{N}, \partial \mathbb{R}_{+}^{N}\right)$ by Lemma 9.1 of [5]. To complete the proof it is then enough to conveniently choose $\left(\sigma_{n}\right)_{n},\left(\tau_{n}\right)_{n}$ in such a way that

$$
\left.\left.u_{\sigma_{n}, \tau_{n}}\right|_{\partial \mathbb{R}_{+}^{N}} \rightarrow u\right|_{\partial \mathbb{R}_{+}^{N}} \quad \text { in } W^{\ell, p}\left(\partial \mathbb{R}_{+}^{N}\right) \quad \text { as } n \rightarrow \infty .
$$

To prove (6) we first note that $W^{k, p}\left(\mathbb{R}^{N}\right)$ is algebraically and topologically isomorphic to its vectorial version $X^{k, p}$ defined in (5), endowed with the standard norm

$$
\|\vec{u}\|_{X^{k, p}}=\left(\sum_{j=0}^{k}\|u\|_{W^{j, p}\left(\mathbb{R} ; W^{k-j}\left(\mathbb{R}^{N-1}\right)\right)}^{p}\right)^{1 / p} \quad \text { for any } \vec{u} \in X^{k, p},
$$

via the isomorphism which associates to each $u \in W^{k, p}\left(\mathbb{R}^{N}\right)$ its vector-valued version $\vec{u}$ defined by

$$
\vec{u}\left(x_{N}\right)=u\left(\cdot, x_{N}\right) \quad \text { for all } x_{N} \in \mathbb{R} .
$$

This result, which is well-known (see for example [1, Example 7.34] or [7, Example 3, p.490] in the similar case of $\mathbb{R}_{+}^{N}$ ), can be easily proved. Indeed the case $k=1$ follows by the general theory of vector-valued Sobolev functions (see [6, Appendix A]) and the extension of the generalized Leibnitz formula [8, Theorem 3, p. $303]$ to the duality product in Banach spaces and to $1 \leq p<\infty$. The general case then follows by induction.

Furthermore, $\left.u\right|_{\partial \mathbb{R}_{+}^{N}}=\vec{u}(0)$ for any $u \in W^{k, p}\left(\mathbb{R}^{N}\right)$ thanks to the identification of $W^{k, p}\left(\mathbb{R}^{N-1}\right)$ with $W^{k, p}\left(\partial \mathbb{R}_{+}^{N}\right)$. Actually, the equality $\left.u\right|_{\partial \mathbb{R}_{+}^{N}}=\vec{u}(0)$ is true for continuous functions and then by density in the entire $W^{k, p}\left(\mathbb{R}^{N}\right)$. Hence $W^{k, \ell, p}\left(\mathbb{R}^{N}, \partial \mathbb{R}_{+}^{N}\right)$ is isomorphic by the same identification to its vectorial version

$$
X^{k, \ell, p}=\left\{(\vec{u}, v) \in X^{k, p} \times W^{\ell, p}\left(\mathbb{R}^{N-1}\right): v=\vec{u}(0)\right\}
$$


endowed with the product norm

$$
\|\vec{u}\|_{X^{k, \ell, p}}=\left(\|\vec{u}\|_{X^{k, p}}^{p}+\|\vec{u}(0)\|_{W^{\ell, p}\left(\mathbb{R}^{N-1}\right)}^{p}\right)^{1 / p} .
$$

Consequently, to show 6 is equivalent to prove that

$$
\vec{u}_{\sigma_{n}, \tau_{n}}(0) \rightarrow \vec{u}(0) \quad \text { in } W^{\ell, p}\left(\mathbb{R}^{N-1}\right),
$$

where $\left(\sigma_{n}\right)_{n},\left(\tau_{n}\right)_{n}$ will be chosen later. In order to prove (7) let us denote by $*^{\prime}$ and $*^{\prime \prime}$ the convolution in $\mathbb{R}^{N-1}$ and in $\mathbb{R}$, respectively.

Since $u \in W^{k, \ell, p}\left(\mathbb{R}^{N}, \partial \mathbb{R}_{+}^{N}\right)$, then $\vec{u} \in X^{k, \ell, p}$ and for all $n$ set

$$
\vec{u}_{n}=\rho_{n}^{\prime} * \vec{u} .
$$

Now Proposition 4.20 and Theorem 4.15 of [5] imply that for any $\rho^{\prime} \in C_{c}^{\infty}\left(\mathbb{R}^{N-1}\right)$, any $v \in L^{p}\left(\mathbb{R}^{N-1}\right)$ and any multi-index $\alpha=\left(\alpha_{1}, \ldots, \alpha_{N-1}\right)$

$$
D^{\alpha}\left(\rho^{\prime} *^{\prime} v\right)=\left(D^{\alpha} \rho^{\prime}\right) *^{\prime} v,
$$

so that $\rho^{\prime} *^{\prime} v \in W^{\ell, p}\left(\mathbb{R}^{N-1}\right)$ and

$$
\left\|D^{\alpha}\left(\rho^{\prime} *^{\prime} v\right)\right\|_{p} \leq\left\|D^{\alpha} \rho^{\prime}\right\|_{1}\|v\|_{p},
$$

whenever $|\alpha| \leq \ell$. Consequently, the linear operator $v \mapsto \rho^{\prime} *^{\prime} v$ is bounded from $L^{p}\left(\mathbb{R}^{N-1}\right)$ into $W^{\ell, p}\left(\mathbb{R}^{N-1}\right)$.

The continuity of the embeddings $X^{k, p} \hookrightarrow W^{1, p}\left(\mathbb{R} ; L^{p}\left(\mathbb{R}^{N-1}\right)\right) \hookrightarrow C_{b}\left(\mathbb{R} ; L^{p}\left(\mathbb{R}^{N-1}\right)\right)$ and the fact that $\vec{u} \in X^{k, p}$ imply that $\vec{u}_{n} \in C\left(\mathbb{R} ; W^{\ell, p}\left(\mathbb{R}^{N-1}\right)\right)$. Now $\vec{u}(0)=\left.u\right|_{\partial \mathbb{R}_{+}^{N}}$ is in $W^{\ell, p}\left(\mathbb{R}^{N-1}\right)$ so that as $n \rightarrow \infty$

$$
\vec{u}_{n}(0) \rightarrow \vec{u}(0) \quad \text { in } W^{\ell, p}\left(\mathbb{R}^{N-1}\right) .
$$

Fubini's theorem yields for a.e. $x=\left(x^{\prime}, x_{N}\right) \in \mathbb{R}^{N}$ that

$$
\begin{aligned}
u_{m, n}(x) & =\int_{\mathbb{R}^{N}} \rho_{m}^{\prime}\left(x^{\prime}-y^{\prime}\right) \rho_{n}^{\prime \prime}\left(x_{N}-y_{N}\right) u\left(y^{\prime}, y_{N}\right) d y^{\prime} d y_{N} \\
& =\int_{\mathbb{R}_{n}} \rho_{n}^{\prime \prime}\left(x_{N}-y_{N}\right)\left(\left(\rho_{m}^{\prime} *^{\prime} \vec{u}\right)\left(x^{\prime}\right)\right)\left(y_{N}\right) d y_{N} \\
& =\left(\rho_{n}^{\prime \prime} *^{\prime \prime}\left(\rho_{m}^{\prime} *^{\prime} \vec{u}\right)\left(x^{\prime}\right)\right)\left(x_{N}\right) .
\end{aligned}
$$

Hence $\vec{u}_{m, n}=\rho_{n}^{\prime \prime} *^{\prime \prime}\left(\rho_{m}^{\prime} *^{\prime} \vec{u}\right)=\rho_{n}^{\prime \prime} *^{\prime \prime} \vec{u}_{n}$. Since $\vec{u}_{n} \in C\left(\mathbb{R} ; W^{\ell, p}\left(\mathbb{R}^{N-1}\right)\right.$ the trivial extension to the vectorial case of well-known properties of regularization, that is of [5. Proposition 4.2], yield that for $m$ fixed $\vec{u}_{m, n} \rightarrow \vec{u}_{m}$ in $W^{\ell, p}\left(\mathbb{R}^{N-1}\right)$ as $n \rightarrow \infty$, uniformly on compact sets of $\mathbb{R}$. Consequently,

$$
\vec{u}_{m, n}(0) \rightarrow \vec{u}_{m}(0) \quad \text { as } n \rightarrow \infty \text {. }
$$

Combining (8) with (9) for $\sigma_{n}=2 n$ we get that $\vec{u}_{2 n}(0) \rightarrow \vec{u}(0)$ in $W^{\ell, p}\left(\mathbb{R}^{N-1}\right)$ and that for any $n$ there exists $\tau_{n} \geq 2 n$, with $\left(\tau_{n}\right)_{n}$ strictly increasing, such that

$$
\left\|\vec{u}_{2 n, \tau_{n}}(0)-\vec{u}_{2 n}(0)\right\|_{W^{\ell, p}\left(\mathbb{R}^{N-1}\right)}<1 / n \text {. }
$$


Thus, $\vec{u}_{2 n, \tau_{n}}(0) \rightarrow \vec{u}(0)$ in $W^{\ell, p}\left(\mathbb{R}^{N-1}\right)$, that is (7) holds. Finally this completes the proof.

Proof of Theorem 1. Let us start by fixing some usual notation. We set

$$
Q=B_{1}\left(\mathbb{R}^{N-1}\right) \times(-1,1), \quad Q_{+}=Q \cap \mathbb{R}_{+}^{N}, \quad Q_{0}=Q \cap \partial \mathbb{R}_{+}^{N} .
$$

Moreover we shall denote by $u_{\mid \Gamma_{i}}$ the restriction of $u_{\mid \Gamma}$ to $\Gamma_{i}$ for $i=0,1$. From the assumption that $\overline{\Gamma_{0}} \cap \overline{\Gamma_{1}}=\emptyset$ it follows that $\Gamma_{0}$ and $\Gamma_{1}$ are compact. Then, using the definition of $C^{m}$ regular open set, see Chapter 9 of [5], there are open subsets $V_{1}, \ldots, V_{r}$, $V_{r+1}, \ldots, V_{s}$ in $\mathbb{R}^{N}$ such that

$\Gamma_{0} \subseteq \bigcup_{j=1}^{r} V_{j}, \quad \Gamma_{1} \subseteq \bigcup_{j=r+1}^{s} V_{j}, \quad V_{j} \cap \Gamma_{1}=\emptyset$ if $j=1, \ldots, r, \quad V_{j} \cap \Gamma_{0}=\emptyset$ if $j=r+1, \ldots, s$, and bijiective maps $H_{j}: Q \rightarrow V_{j}, j=1, \ldots, s$, such that

$$
\begin{gathered}
H_{j} \in C^{m}(\bar{Q}), \quad H_{j}^{-1} \in C^{m}\left(\overline{V_{j}}\right), \quad H_{j}\left(Q_{+}\right)=V_{j} \cap \Omega, \quad H_{j}\left(Q_{0}\right)=V_{j} \cap \Gamma_{0}, j=1, \ldots, r, \\
H_{j}\left(Q_{0}\right)=V_{j} \cap \Gamma_{1}, j=r+1, \ldots, s .
\end{gathered}
$$

Moreover, from Lemma 9.3 of [5], see also [11, Theorem C.21] for a proof, there are functions $\theta_{0}, \ldots, \theta_{s} \in C^{\infty}\left(\mathbb{R}^{N}\right)$ such that

$$
0 \leq \theta_{j} \leq 1, \quad \sum_{j=0}^{s} \theta_{j}=1 \text { in } \mathbb{R}^{N},\left.\quad \theta_{0}\right|_{\Omega} \in C_{c}^{\infty}(\Omega), \quad \operatorname{supp} \theta_{j} \subset \subset V_{j} \text { for } j=1, \ldots, s .
$$

Now let $u \in W^{k, \ell, p}(\Omega, \Gamma)$. Since $u \in W^{k, p}(\Omega)$ and $\Omega$ is $C^{m}$ regular we can extend it to $\tilde{u} \in W^{k, p}\left(\mathbb{R}^{N}\right)$ by Theorem 4.26 of [1]. Put $u_{j}=\theta_{j} \tilde{u} \in W^{k, p}\left(\mathbb{R}^{N}\right)$, so that

$$
\begin{gathered}
\tilde{u}=\sum_{j=0}^{s} u_{j} \text { in } \mathbb{R}^{N}, \quad u_{\mid \Gamma_{0}}=\sum_{j=0}^{r} u_{j \mid \Gamma_{0}}, \quad u_{\mid \Gamma_{1}}=\sum_{j=r+1}^{s} u_{j \mid \Gamma_{1}}, \\
\quad \operatorname{supp} u_{0} \subset \subset \Omega, \quad \operatorname{supp} u_{j} \subset \subset V_{j} \text { for } j=1, \ldots, s .
\end{gathered}
$$

We shall show that each $u_{j}, j=0, \ldots, s$, can be approximated by elements of $Y$ in the $W_{\Gamma_{0}}^{k, \ell, p}(\Omega ; \Gamma)$ norm. For $j=0$ there is nothing to prove since by Lemma 3.15 of [1] there is a sequence $\left(\psi_{n}^{0}\right)_{n}$ in $C_{c}^{\infty}(\Omega)$ such that $\left.\psi_{n}^{0} \rightarrow u_{0}\right|_{\Omega}$ in $W^{k, p}(\Omega)$ and $\psi_{n \mid \Gamma}^{0}=u_{0 \mid \Gamma}=0$ for all $n$. Indeed, $\psi_{n \mid \Gamma}^{0}=0$ by [11, Theorem 15.29 p. 475] and so $u_{0 \mid \Gamma}=0$ by the continuity of the Trace Operator and the fact that $\left.\psi_{n}^{0} \rightarrow u_{0}\right|_{\Omega}$ in $W^{k, p}(\Omega)$.

Fix now $j=1, \ldots, s$. By Theorem 3.41 of [2] on the stability of Sobolev spaces with respect to coordinate transformations the linear operator $v \mapsto v \cdot H_{j}$ is bounded from $W^{k, p}(\Omega)$ onto $W^{k, p}(\Omega)$, with bounded inverse. Hence $u_{j} \cdot H_{j} \in W^{k, p}(Q)$. Moreover, $\operatorname{supp}\left(u_{j} \cdot H_{j}\right) \subset \subset Q$, since $\operatorname{supp} u_{j} \subset \subset V_{j}$. Then Lemma 3.15 of [1] and Theorem 5.29 of [2] yield that $u_{j} \cdot H_{j} \in W_{0}^{k, p}(Q)$ and so its trivial extension $v_{j}=\widetilde{u_{j} \cdot H_{j}}$ to the whole of $\mathbb{R}^{N}$ belongs to $W^{k, p}\left(\mathbb{R}^{N}\right)$. 
By the definition of Sobolev spaces on $\Gamma$ we have $\left.\widetilde{u_{j} \cdot H_{j}}\right|_{\partial \mathbb{R}_{+}^{N}} \in W^{\ell, p}\left(\mathbb{R}^{N-1}\right)$. Thus $v_{j} \in W^{k, \ell, p}\left(\mathbb{R}^{N} ; \partial \mathbb{R}_{+}^{N}\right)$. By Theorem 2 there exists a sequence

$$
\left(\varphi_{j, n}\right)_{n} \quad \text { in } \quad W^{k, \ell, p}\left(\mathbb{R}^{N}, \partial \mathbb{R}_{+}^{N}\right) \cap C^{\infty}\left(\mathbb{R}^{N}\right)
$$

such that $\varphi_{j, n} \rightarrow v_{j}$ in $W^{k, \ell, p}\left(\mathbb{R}^{N} ; \partial \mathbb{R}_{+}^{N}\right)$ as $n \rightarrow \infty$ and $\operatorname{supp} \varphi_{j, n} \subseteq \operatorname{supp} v_{j}+\overline{B_{1 / n}\left(\mathbb{R}^{N}\right)}$. But supp $v_{j} \subset \subset Q$ so that there exists $r_{j} \in(0,1)$ such that $\varphi_{j, n} \in C_{c}^{\infty}(Q)$ and supp $\varphi_{j, n} \subseteq$ $\overline{B_{r_{j}}\left(\mathbb{R}^{N-1}\right)} \times\left[-r_{j}, r_{j}\right]$ for $n$ sufficiently large.

Let us now distinguish two cases: $j \in\{r+1, \ldots, s\}$ and $j \in\{1, \ldots, r\}$. When $j \in\{r+1, \ldots, s\}$ we set $\psi_{j, n}=\varphi_{j, n} \cdot H_{j}^{-1} \in C_{c}^{m}\left(V_{j}\right)$. An application of Theorem 3.41 of [2] yields that $\psi_{j, n} \rightarrow u_{j}$ in $W^{k, p}\left(\mathbb{R}^{N}\right)$ as $n \rightarrow \infty$, while $\psi_{j, n \mid \Gamma} \rightarrow u_{j \mid \Gamma}$ in $W^{\ell, p}(\Gamma)$ by the definition of $W^{\ell, p}(\Gamma)$. But $\psi_{j, n} \in W_{\Gamma_{0}}^{k, \ell, p}(\Omega ; \Gamma)$, since in this case $V_{j} \cap \Gamma_{0}=\emptyset$. Thus $\psi_{j, n} \rightarrow u_{j}$ in $W_{\Gamma_{0}}^{k, \ell, p}(\Omega ; \Gamma)$, as stated.

When $j \in\{1, \ldots, r\}$ we do not know any longer that $\varphi_{j, n} \cdot H_{j}^{-1}$ vanishes on $\Gamma_{0}$, so that we need to conveniently modify $\varphi_{j, n}$. To this aim we introduce a cut-off function $\xi \in C^{\infty}(\mathbb{R})$ such that $\xi(0)=1$ and $\operatorname{supp} \xi \subseteq\left[-r_{j}, r_{j}\right]$. The linear operator $L: W^{k, p}\left(\mathbb{R}^{N-1}\right) \rightarrow W^{k, p}\left(\mathbb{R}^{N}\right)$ defined by $(L w)(x)=w\left(x^{\prime}\right) \xi\left(x_{N}\right)$ for all $w \in C^{\infty}\left(\mathbb{R}^{N-1}\right)$ is bounded and by density $L$ is well defined in the entire $W^{k, p}\left(\mathbb{R}^{N-1}\right)$. Clearly, $L w$ is of class $C^{\infty}\left(\mathbb{R}^{N}\right)$ and $\left.L w\right|_{\partial \mathbb{R}_{+}^{N}}=w$ for all $w \in C^{\infty}\left(\mathbb{R}^{N-1}\right)$, while $L w \in C_{c}^{\infty}\left(\mathbb{R}^{N}\right)$ for all $w \in C_{c}^{\infty}\left(B_{1}\left(\mathbb{R}^{N-1}\right)\right)$. Set $\chi_{j, n}=L \varphi_{j, n \mid \partial \mathbb{R}_{+}^{N}}$, so that $\chi_{j, n \mid \partial \mathbb{R}_{+}^{N}}=\varphi_{j, n \mid \partial \mathbb{R}_{+}^{N}}$ and moreover $\chi_{j, n \mid \partial \mathbb{R}_{+}^{N}} \rightarrow 0$ in $W^{\ell, p}\left(\mathbb{R}^{N-1}\right)$ and so in $W^{k, p}\left(\mathbb{R}^{N-1}\right)$, since $k \leq \ell$. The fact that $L$ is bounded implies that $\chi_{j, n} \rightarrow 0$ in $W^{k, p}\left(\mathbb{R}^{N}\right)$. Put $\tilde{\varphi}_{j, n}=\varphi_{j, n}-\chi_{j, n}$. Then $\tilde{\varphi}_{j, n} \in C_{c}^{\infty}\left(\mathbb{R}^{N}\right)$ for all $n$ and $\tilde{\varphi}_{j, n} \rightarrow v_{j}$ in $W^{k, \ell, p}\left(\mathbb{R}^{N} ; \partial \mathbb{R}_{+}^{N}\right)$, supp $\tilde{\varphi}_{j, n} \subset \subset Q$ and finally $\tilde{\varphi}_{j, n}=0$ on $\partial \mathbb{R}_{+}^{N}$. Set $\psi_{j, n}=\tilde{\varphi}_{j, n} \cdot H_{j}^{-1} \in C_{c}^{m}\left(V_{j}\right)$. Consequently, as in the previous case, $\psi_{j, n} \rightarrow w_{j}$ in $W_{\Gamma_{0}}^{k, \ell, p}(\Omega ; \Gamma)$.

Lastly, the previous steps show that $\psi_{n}=\sum_{j=0}^{s} \psi_{j, n}$, which is in $Y$, converges to $u$ in $W_{\Gamma_{0}}^{k, \ell, p}(\Omega ; \Gamma)$. This completes the proof.

\section{References}

1. Adams, R.A.: Sobolev spaces. Academic Press, New York-London (1975). Pure and Applied Mathematics, Vol. 65

2. Adams, R.A., Fournier, J.J.F.: Sobolev spaces, Pure and Applied Mathematics (Amsterdam), vol. 140, second edn. Elsevier/Academic Press, Amsterdam (2003)

3. Bardaro, C., Candeloro, D.: Approximation theorems for the multiple integral of the calculus of variations. Rend. Circ. Mat. Palermo (2) 30(1), 63-82 (1981)

4. Brezis, H.: Analyse fonctionnelle. Masson, Paris (1983). Théorie et applications.

5. Brezis, H.: Functional analysis, Sobolev spaces and partial differential equations. Universitext. Springer, New York (2011)

6. Brezis, H., Cazenave, T.: Nonlinear evolution equations. Unpublished

7. Dautray, R., Lions, J.L.: Mathematical analysis and numerical methods for science and technology. Vol. 5. Springer-Verlag, Berlin (1992)

8. Evans, L.C.: Partial differential equations, Graduate Studies in Mathematics, vol. 19, second edn. American Mathematical Society, Providence, RI (2010)

9. Grisvard, P.: Elliptic problems in nonsmooth domains, Monographs and Studies in Mathematics, vol. 24. Pitman (Advanced Publishing Program), Boston, MA (1985) 
10. Kashiwabara, T., Colciago, C.M., Dedè, L., Quarteroni, A.: Well-Posedness, Regularity, and Convergence Analysis of the Finite Element Approximation of a Generalized Robin Boundary Value Problem. SIAM J. Numer. Anal. 53(1), 105-126 (2015)

11. Leoni, G.: A first course in Sobolev spaces, Graduate Studies in Mathematics, vol. 181, second edn. American Mathematical Society, Providence, RI (2017)

12. Lions, J.L.: Lectures on elliptic partial differential equations. In: Tata Institute of Fundamental Research Lectures on Mathematics, No. 10, pp. iii+130+vi. Tata Institute of Fundamental Research, Bombay (1967)

13. Lions, J.L., Magenes, E.: Problèmes aux limites non homogènes et applications. Vol. 1. Travaux et Recherches Mathématiques, No. 17. Dunod, Paris (1968)

14. Maz'ja, V.G.: Sobolev spaces. Springer Series in Soviet Mathematics. Springer-Verlag, Berlin (1985). Translated from the Russian by T. O. Shaposhnikova

15. Meyers, N.G., Serrin, J.: $H=W$. Proc. Nat. Acad. Sci. U.S.A. 51, 1055-1056 (1964)

16. Nečas, J.: Direct methods in the theory of elliptic equations. Springer Monographs in Mathematics. Springer, Heidelberg (2012)

17. Triebel, H.: Interpolation theory, function spaces, differential operators. North-Holland, Amsterdam (1978)

18. Vitillaro, E.: On the Wave Equation with Hyperbolic Dynamical Boundary Conditions, Interior and Boundary Damping and Source. Arch. Ration. Mech. Anal. 223(3), 1183-1237 (2017). DOI 10.1007/ s00205-016-1055-2

19. Vitillaro, E.: On the wave equation with hyperbolic dynamical boundary conditions, interior and boundary damping and supercritical sources. J. Differential Equations 265(10), 4873-4941 (2018)

20. Ziemer, W.P.: Weakly differentiable functions, Graduate Texts in Mathematics, vol. 120. SpringerVerlag, New York (1989). Sobolev spaces and functions of bounded variation 